\title{
Sur les sous-groupes fermés connexes d'un groupe de Lie clos
}

\section{Doctoral Thesis}

Author(s):

Siebenthal, Jean <<de>>

Publication date:

1951

Permanent link:

https://doi.org/10.3929/ethz-a-000092445

Rights / license:

In Copyright - Non-Commercial Use Permitted 


\section{SUR LES}

\section{SOUS-GROUPES FERMES CONNEXES D'UN GROUPE DE LIE CLOS}

\section{TH H S S E}

PRÉSENTÉE A L'ÉCOLE POLYTECHNIQUE FÉDÉRALE, ZURICH POUR L'OBTENTION DU GRADE DE DOCTELR ASS SCTENCES MATHEMATIQUES

PAR

\section{JEAN DE SIEB ENTHAL}

DE SAANEN (BERNE)

RAPPORTEUR: PROF. DR E. STIEFEL

CORAPPORTEUR: PROF. DR H. HOPF

\section{$\begin{array}{llll}1 & 9 & 5 & 1\end{array}$}

O R E L L F ÜS S I A R T S G A P H I Q UES S. A. Z U R I C H 


\title{
Sur les sous-groupes fermés connexes d'un groupe de Lie clos
}

\author{
Par Jean De Siebenthal, Lausanne
}

\section{Introduction}

1. L'étude d'un groupe $G$ ne peut s'achever si l'on n'en connaît pas les sous-groupes. Lorsque $G$ est un groupe de Lie, on peut se proposer de rechercher tous les sous-groupes continus connexes de ce groupe; en se plaçant au point de vue local, S. Lie a montré qu'on peut se ramener à un problème purement algébrique, théoriquement résoluble dès qu'on connaît les constantes de structure de $G^{1}$ ). De façon plus précise, si $X_{1}, X_{2}, \ldots, X_{n}$ est une base de l'anneau $R(G)$ du groupe $G$, avec la loi de composition $\left[X_{i}, X_{j}\right]=\sum_{k=1}^{n} c_{i j k} X_{k}$, on peut trouver tous les sousgroupes connexes de $G$ qui sont des groupes de Lie au sens local en déterminant tous les sous-anneaux de $R(G)$, e'est-à-dire tous les sousespaces $R$ de $R(G)$ tels que $X, Y \in R$ entraînent $[X, Y] \in R$. Ainsi posé, le problème n'est pas facile à aborder.

Deux simplifications s'imposent d'emblée : d'abord, il suffit de chercher un sous-groupe dans chaque classe de sous-groupes conjugués; ensuite, on peut se borner à la recherche des plus grands sous-groupes de $G$, cette expression désignant ici les sous-groupes propres connexes qui ne sont pas contenus dans un autre sous-groupe propre connexe de $G$.

2. Le problème envisagé est le suivant

(a) Déterminer les sous-groupes fermés connexes d'un groupe de Lie clos (ou compact).

Tout sous-groupe continu d'un groupe de Lie étant un groupe de $\mathrm{Lie}^{2}$ ), on peut appliquer la méthode algébrique décrite ci-dessus, et déterminer

1) S. Lie, Theorie der Transformationsgruppen, t. I, p. 209 (Teubner, Leipzig 1888).

2) E. Cartan, La théorie des groupesfinis et continus et l'analysis situs (Mém. Sc. Math., t. 42, 1930, p. 22). 
tous les sous-anneaux $R$ de $R(G)$. Cela fait, il reste à examiner si le sous-groupe fermé connexe engendré par $R$ a même dimension que $R$ (voir chapitre II, § 4).

Le problème algébrique basé sur les constantes de structure peut à son tour être réduit à un problème plus simple; en effet, le groupe clos $G$ supposé semi-simple est déterminé localement par ses paramètres angulaires ${ }^{3}$ ), qui sont $2 m$ formes linéaires $\pm \mu_{1}(x), \ldots, \pm \mu_{m}(x), x$ étant un point d'un espace euclidien $R^{l}$ à $l$ dimensions, dont la métrique est donnée par la forme quadratique $\Sigma\left[\mu_{i}(x)\right]^{2}$; l'entier $l$ est le rang du groupe. La figure constituée par l'espace $R^{l}$ et par les plans $\mu_{i}(x) \equiv 0$ (mod. 1) est le diagramme de $G^{10}$ ); le diagramme $R^{h}$ d'un sous-groupe est alors un sous-diagramme du précédent. A ce point de vue, le problème s'énonce ainsi

(b) Trouver les conditions nécessaires et suffisantes pour qu'un sousespace linéaire de $R^{l}$ soit le support d'un sous-diagramme représentant un sous-groupe fermé connexe.

Il convient de remarquer qu'un même sous-diagramme peut représenter plusieurs sous-groupes, naturellement isomorphes, dont il serait intéressant de savoir s'ils sont conjugués. Le groupe $G$ n'est pas distingué des groupes clos qui lui sont localement isomorphes.

On peut donner au problème (b) un aspect plus intuitif ; soit $\vec{\mu}_{i}$ le vecteur de $R^{l}$ défini par $\mu_{i}(x)=-\vec{\mu}_{i} \cdot \vec{x}$. Il existe dans l'ensemble $\pm \vec{\mu}_{1}, \pm \vec{\mu}_{2}, \ldots, \pm \vec{\mu}_{m} l$ vecteurs fondamentaux $\vec{\varphi}_{1}, \ldots, \vec{\varphi}_{l}\left(\vec{\varphi}_{i} \cdot \vec{\varphi}_{j} \leq 0\right)$ qui déterminent le diagramme et l'anneau $R(G)$; ils vérifient certaines conditions simples énoncées par van der Waerden $\left.{ }^{3}\right)$. Les $h$ vecteurs $\vec{\varrho}_{1}, \ldots, \vec{\varrho}_{h}$, fondamentaux pour le sous-groupe étudié, sont des combinaisons linéaires de $\vec{\varphi}_{1}, \ldots, \vec{\varphi}_{l}$ qui vérifient encore les conditions citées. L'énoncé correspondant est

(c) Soient $\vec{\varphi}_{1}, \vec{\varphi}_{2}, \ldots, \vec{\varphi}_{l} l$ vecteurs fondamentaux du groupe clos $G$; trouver $h$ vecteurs $\vec{\varrho}_{1}, \ldots, \vec{\varrho}_{h}$ ayant les propriétés suivantes

(1) $\vec{\varrho}_{1}, \ldots, \vec{\varrho}_{h}$ sont des combinaisons linéaires de $\vec{\varphi}_{1}, \ldots, \vec{\varphi}_{l}$.

(2) $\vec{\varrho}_{1}, \ldots, \vec{\varrho}_{h}$ forment la figure fondamentale d'un groupe clos.

$\left.{ }^{3}\right)$ van der Waerden, Die Klassifikation der einfachen Lieschen Gruppen (Math. Zeitschr., t. 37, 1933, p. 448). 
(3) Il existe dans $G$ un sous-groupe fermé connexe admettant $\vec{\varrho}_{1}, \ldots, \vec{\varrho}_{h}$ comme figure fondamentale.

3. A ma connaissance, le problème posé en (a) n'a jamais été abordé systématiquement au sens indiqué en (b) ou (c). Cela n'empêche nullement l'existence d'un grand nombre d'énoncés sur les sous-groupes des groupes de Lie clos ou non; ces énoncés ouvrent diverses voies :

Les sous-groupes commutatifs maximums d'un groupe clos $G$ ont fait l'objet d'études précises, par E. Cartan $^{4}$ ) au point de vue infinitésimal, et par H.Hopf ${ }^{5}$ ) au point de vue global. La notion de sous-groupe commutatif maximum est d'ailleurs à la base de la représentation de $G$ par son diagramme.

Les sous-groupes d'isotropie $G_{1}$ des espaces symétriques clos ont été étudiés de façon systématique par $E$. Cartan $^{6}$ ), en partant des automorphismes involutifs de $G$ dont ces sous-groupes sont caractéristiques. $\mathrm{Je}$ signale encore que $A$. Malcev $^{7}$ ) a déterminé les sous-groupes semisimples des groupes de Lie complexes, à l'aide des représentations linéaires de ces groupes.

La résolution complète du problème posé en (a) permettrait par exemple de faire une étude d'ensemble des espaces homogènes clos $G / G_{1}$, et de savoir en particulier si la classe des espaces symétriques clos contient la plupart des premiers ou non. Un autre problème pourrait être abordé : étant donné un groupe clos $G$ et un sous-groupe fermé connexe $G_{1}$ de $G$, quand $G_{1}$ est-il homologue à zéro dans $G$ (au sens de la topologie combinatoire)? On sait d'après E. Cartan qu'un sous-groupe simple à trois paramètres n'est jamais homologue à zéro; il en est de même de tout sous-groupe invariant fermé connexe.

Dans un autre ordre d'idées, je mentionne qu'on peut démontrer le théorème suivant: il n'y a dans le diagramme $D(G)$ d'un groupe clos $G$ qu'un nombre fini de sous-diagrammes $D\left(G_{1}\right)$. De plus, toute chaîne de sous-groupes $G_{1} \subset G_{2} \subset \cdots<G$ peut être représentée dans $D(G)$ par une chaîne $D\left(G_{1}\right) \subset D\left(G_{2}\right) \subset \cdots \subset D(G)$; l'ensemble de ces chaînes est encore fini. Il serait peut-être intéressant d'étudier ces ensembles finis, et de savoir de quelles structures on pourrait les munir.

4) E. Cartan, La géométrie des groupes simples (Annali di Mat., t. 4, 1927, p. 212 à 214).

5) H. Hopf, Ober den Rang geschlossener Liescher Gruppen (Comment. Math. Helv., t. 13, 1940, p. 119-143).

6) E.Cartan, Sur certaines formes riemanniennes remarquables des géométries à groupefondamen tal simple (Ann. Ec. Norm. (3) XLIV, 1927, p. 345-467).

7) A. Malcev, On semi -simple subgroups of Lie groups (Bull. Acad. Sci. U. R. S. S., Sér. Math. 8, p. 143-174, 1944). D'après le résumé: Math. Rev., t. 6, p. 146. 
4. Une note déjà parue indique que le problème (a) est résolu dans le cas où le rang du sous-groupe est égal au rang du groupe $\left.{ }^{8}\right)$. D'ailleurs, ce même problème est aussi résolu lorsque le rang du sous-groupe est égal à un ${ }^{9}$ ). Les pages qui suivent n'apportent pas la solution complète; elles présentent un certain nombre de résultats généraux, appliqués à un cas particulier important.

Le chapitre I pose les notions classiques relatives à un groupe clos $G$, d'après $E$. Stiefel $\left.{ }^{10}\right)$ : diagramme, ensemble $\Sigma(G)$ des paramètres angulaires de $G$, groupe fini $\Phi(G)$ des automorphismes intérieurs de $G$ qui laissent invariant un sous-groupe commutatif maximum donné ; j'introduis de plus la notion de diagonale du diagramme: $\varphi_{1}=\varphi_{2}=\cdots$ $=\varphi_{k}=0 ; \quad \varphi_{k+1}=\cdots=\varphi_{l} \neq 0$, et la figure de Schläfli $\mathfrak{F}(G)$ qui représente la figure $\vec{\varphi}_{1}, \vec{\varphi}_{2}, \ldots, \vec{\varphi}_{l}$ par $l$ points reliés par certains traits.

Cela posé, j'associe (chapitre II) au groupe $G$ et au sous-groupe $G_{1}$ un tableau

$$
\mathrm{I}\left\{\begin{array}{l}
\varrho_{1}: \alpha_{1}, \ldots, \alpha_{n_{1}} \\
\varrho_{2}: \beta_{1}, \ldots, \beta_{n_{2}} \\
\ldots \\
\varrho_{h}: \gamma_{1}, \ldots, \gamma_{n_{h}}
\end{array}\right.
$$

dans lequel $\varrho_{1}, \varrho_{2}, \ldots, \varrho_{h}$ sont $h$ paramètres angulaires fondamentaux $\operatorname{de} G ; \alpha_{1}, \alpha_{2}, \ldots, \alpha_{n_{1}}$ sont les formes de $\Sigma(G)$ qui se réduisent à $\varrho_{1}$ dans $R^{h}$... Le tableau I est le principal objet de cette étude $(\S 5,6,7)$. Dans ce même chapitre, je montre qu'il suffit de considérer les sous-groupes de $G$ qui ne sont pas contenus dans un sous-groupe propre de rang maximum de $G$; ce sont les sous-groupes $(H)$. Alors, tout $\omega \in \Sigma(G)$ est une combinaison linéaire à coefficients entiers des formes du tableau I.

Les chapitres III et IV contiennent l'étude des sous-groupes remarquables dont le tableau $\mathrm{I}$ contient $l$ paramètres angulaires fondamentaux et ceux-là seulement (sous-groupes $\left.(H)_{0}\right)$. La figure de Schläfli $\mathfrak{F}(G)$ doit être appliquée d'une certaine façon sur ce tableau $(\$ 9,10,11)$. La discussion fournit les résultats suivants: si le rang $h$ de $G_{1}$ est au moins égal à 2 , les seuls sous-groupes $(H)_{0}$ des groupes clos simples sont les sous-groupes caractéristiques d'automorphismes involutifs externes ${ }^{11}$ ),

8) A. Borel et J. de Siebenthal, C. R. Acad. Sc., t. 226, p. 1662-1664; voir aussi : Comment. Math. Helv., t. 23, 1949, p. 200-221.

$\left.{ }^{9}\right)$ J. de Siebenthal, C. R. Acad. Sc., t. 230, 1950, p. 910-912.

10) E. Stiefel, Comment. Math. Helv., t. 14, 1942, p. 350-380.

11) E. Cartan, cf. note 6 . 
à trois exceptions près: $G_{2} \subset B_{3}, G_{2}\left(A_{6}, G_{2}\left(D_{4}{ }^{12}\right)\right.$. Les inclusions $C_{4} \subset E_{6}$ et $D_{h} \subset A_{2 h-1}$ ne rentrent pas dans le cas étudié.

Le cas où le rang du sous-groupe $(H)_{0}$ est égal à un est très différent : tout groupe clos $G$ non abélien contient un sous-groupe $(H)_{0}$ de rang un, dit sous-groupe principal. Ce sous-groupe admet une définition indépendante du diagramme : un sous-groupe $(H)$ de rang un est dit principal s'il contient un élément régulier dans $G$. Lorsque $G$ est de l'un des types $B_{l}(l>3), C_{l}(l \geq 2), F_{4}, E_{7}, E_{8}$, le sous-groupe principal est toujours maximum.

\section{CHAPITRE I}

\section{Groupes clos}

\section{\$1. Diagramme d'un groupe clos}

1. Torö̈des maximums. Soit $Q$ un groupe de Lie clos connexe; du point de vue topologique, $G$ est un espace de Hausdorff bicompact ${ }^{13}$ ), ou compact au sehs de $\mathrm{N}$. Bourbaki. Pour étudier $G$, il convient de mettre en évidence les sous-groupes commutatifs maximums de $G$, ou toroïdes maximums $T$ de $G$; ils sont tous connexes et ont la même dimension (chaque $T$ est un produit direct de $l$ cercles); l'entier $l$ est le rang du groupe $G$. On a les propriétés suivantes $\left.{ }^{14}\right)$ :

(a) Etant donné un élément $a \in G$, il existe un torö̈de maximum $T$ qui contient a .

(b) Etant donnés deux toroüdes maximums $T$ et $T^{\prime}$, il existe un élément $b \in G$ tel que $b T b^{-1}=T^{\prime}$ (E. Cartan).

(c) Si a est un élément de $G$ échangeable avec tous les éléments d'un torö̈de maximum $T$ de $G$, a appartient $\dot{a} T$.

2. Groupe fini $\Phi(G)$. Le normalisateur $N(T)$ du toroïde maximum $T$ est un sous-groupe de $G$ constitué par un nombre fini de composantes connexes; l'une d'elles est précisément $T$, qui est un sous-groupe invariant de $N(T)$. Soit a un élément de $N(T)$; l'automorphisme intérieur $x \rightarrow a x a^{-1}, x \in G$ induit une transformation de $T$ sur lui-même ; toutes ces transformations forment un groupe fini $\Phi(G)$ isomorphe au groupe quotient $N(T) / T$. A chaque groupe clos $G$ est ainsi associé un groupe fini $\Phi(G)$ de transformations du toroïde $T$.

\footnotetext{
12) Ces inclusions sont bien connues.

13) voir note 2 de l'introduction; cf. No. 9.

${ }^{14}$ ) voir note 5 de l'introduction et $H$. Hopf et H. Samelson (Comment. Math. Helv., t. 13, No 4, Hilfssatz 4).
} 